\title{
A systematic review of the nature of dispensing errors in hospital pharmacies
}

This article was published in the following Dove Press journal:

Integrated Pharmacy Research and Practice

12 January 2016

Number of times this article has been viewed

\section{Khaled Aldhwaihi \\ Fabrizio Schifano \\ Cinzia Pezzolesi \\ Nkiruka Umaru}

Department of Pharmacy, University of Hertfordshire, Hatfield, UK
Correspondence: Khaled Aldhwaihi

Department of Pharmacy, University

of Hertfordshire, College Lane,

Hatfield, ALI0 9NL, UK

Tel +44 I7 07286519

Email kaldhwaihi@gmail.com
Background: Dispensing errors are common in hospital pharmacies. Investigating dispensing errors is important for identifying the factors involved and developing strategies to reduce their occurrence.

Objectives: To review published studies exploring the incidence and types of dispensing errors in hospital pharmacies and factors contributing to these errors.

Methods: Electronic databases including PubMed, Scopus, Ovid, and Web of Science were searched for articles published between January 2000 and January 2015. Inclusion criteria were: studies published in English, and studies investigating type, incidence and factors contributing to dispensing errors in hospital pharmacies. One researcher searched for all relevant published articles, screened all titles and abstracts, and obtained complete articles. A second researcher assessed the titles, abstracts, and complete articles to verify the reliability of the selected articles.

Key findings: Fifteen studies met the inclusion criteria all of which were conducted in just four countries. Reviewing incident reports and direct observation were the main methods used to investigate dispensing errors. Dispensing error rates varied between countries $(0.015 \%-33.5 \%)$ depending on the dispensing system, research method, and classification of dispensing error types. The most frequent dispensing errors reported were dispensing the wrong medicine, dispensing the wrong drug strength, and dispensing the wrong dosage form. The most common factors associated with dispensing errors were: high workload, low staffing, mix-up of look-alike/ sound-alike drugs, lack of knowledge/experience, distractions/interruptions, and communication problems within the dispensary team.

Conclusion: Studies relating to dispensing errors in hospital pharmacies are few in number and have been conducted in just four countries. The majority of these studies focused on the investigation of dispensing error types with no mention of contributing factors or strategies for reducing dispensing errors. Others studies are thus needed to investigate dispensing errors in hospital pharmacies, and a combined approach is recommended to investigate contributing factors associated with dispensing errors and explore strategies for reducing these errors.

Keywords: dispensing errors, medication errors, hospital pharmacy, patient safety, contributing factors

\section{Introduction}

Medication error is one of the most common patient safety incidents reported in hospitals. ${ }^{1,2}$ In England and Wales, 80,000 medication errors were reported to the National Reporting and Learning System (NRLS) by National Health Service organizations between October 1, 2013 and March 31, 2014. ${ }^{3}$ 
Dispensing medication is a complex process that involves more than simply taking medicines from a pharmacy shelf, sticking a label on a pack, and giving this to the patient after containing it. ${ }^{4}$ It is reported that more than a million prescriptions were dispensed in pharmacies in England in $2012^{5}$ and $\sim 4$ billion prescriptions are dispensed every year in the USA. ${ }^{6}$ Dispensing errors are common in hospital pharmacies. In the UK, according to the NRLS, $\sim 17 \%$ of medication errors reported between January and December 2007 were the result of dispensing errors that occurred in general, acute, or community hospitals. ${ }^{7}$ In the USA, Flynn et $\mathrm{al}^{8}$ observed four dispensing errors per day per 250 prescriptions in 50 pharmacies.

The process of medication use in a hospital environment typically consists of several stages: prescribing, transcribing, dispensing, counseling, administration, and monitoring. Depending on the hospital's system, some stages are excluded such as the transcribing stage. Counseling and administration can precede each other depending on the status of the recipient of the drug. Medication errors most commonly occur in the prescribing, dispensing, and administration stages, ${ }^{9-13}$ as these are the three main principle medication use processes. In England and Wales, of the 60,000 medication errors reported to the National Reporting and Learning System (NRLS) between January 2005 and June 2006, $59 \%$ occurred in the administration stage, 17.8\% occurred in the dispensing stage, and $15.7 \%$ occurred in the prescription stage. ${ }^{9}$ Studies conducted on dispensing errors show a high rate of dispensing errors of between $0.04 \%$ and $24 \%$ in community pharmacies. ${ }^{14}$

Some hospital pharmacies collect data regarding dispensing errors identified in the final accuracy check stage (near miss[es]), in order to investigate further; ${ }^{15}$ however, information about data collected as well as the outcomes of such reports are usually limited to the pharmacy department or personnel responsible for collating the data. The lack of information about outcomes of such reports also applies crucially to errors which are identified after the dispensing process. The numbers of dispensing errors noted have remained comparable.

Despite the frequency of dispensing errors in hospitals, less attention has been paid to these in published studies in comparison to prescription and administrative errors. ${ }^{16,17}$ There are a limited number of studies that have reported on dispensing errors in community and hospital pharmacies; however, one review study ${ }^{18}$ was conducted in 2008 to evaluate these studies. The present review focuses on dispensing errors in hospital pharmacies only, as this is a specific angle and does not involve working patterns or systems between the community and hospital pharmacies. Accordingly, it allows the researchers to focus purely on hospital pharmacies, characterized by the dispensing of complex regimens for very ill patients in comparison to community pharmacies and different dispensing systems across different hospitals. This systematic literature review therefore aims to investigate the incidence types and factors associated with dispensing errors in hospital pharmacies, as reported in published literature.

\section{Methods}

The PubMed, Scopus, Ovid, and Web of Science electronic databases were used to identify relevant published articles from January 2000 to January 2015. The keywords used to search for the relevant studies were as follows: Dispensing, Drug(s), Medication, Medicine(s), Error(s), Incident(s), Near miss(es), Mistake(s), Hospital, Secondary care, Inpatient, Outpatient, Pharmacy, Pharmacist, and Dispensary.

\section{Inclusion and exclusion criteria}

"Dispensing error" refers to any error occurring at any dispensing stage in a hospital pharmacy, whether discovered in the pharmacy department or after the medication has left the department. All studies investigating type and/or incidence and/or factors contributing to dispensing errors were included. Studies had to have been undertaken in hospital pharmacies and published in the English language between January 2000 and January 2015.

The researchers excluded any study conducted to identify dispensing errors in community pharmacies or ward stocks or automation dispensing errors. Case reports were not included in this review because they do not reflect the incidence or nature of dispensing errors but rather focus on a specific case. Also excluded were all general medication error studies not specific to dispensing errors as well as conference papers, reviews, opinions, and editorial papers.

\section{Study selection}

Initially, the literature search was conducted by the first author (KA); then, titles were exported from the databases into Endnote X7. All the titles were screened by the first author to identify relevant studies; abstracts were then examined by the first author. Another reviewer (NU) independently reviewed the titles, abstracts, and articles to determine the relevance of studies in terms of meeting the criteria and to exclude irrelevant titles. 


\section{Quality assessment}

The quality of all selected studies was assessed using 12 criteria outlined by Allan and Barker ${ }^{19}$ and modified by Alsulami et $\mathrm{al}^{16}$ and Ghaleb et $\mathrm{al}^{17}$ in order to apply to any type of medication error study. The definition of what constitutes a medication error was changed to a definition of what constitutes a dispensing error. The selected studies had to satisfy a minimum of six criteria from the following list:

1. Aims/objectives of the study clearly stated

2. Definition of what constitutes a dispensing error

3. Error categories specified

4. Error categories defined

5. Presence of a clearly defined denominator

6. Data collection method described clearly

7. Setting in which study conducted described

8. Sampling and calculation of sample size described

9. Reliability and validity measures applied

10. Limitations of study listed

11. Indication of any assumptions made

12. Ethical approval obtained.

\section{Results}

The keyword search resulted in a total of 3,767 studies across all the databases accessed. Duplicate studies were excluded, bringing the total down to 2,929. Following this, article titles and abstracts were reviewed and any irrelevant studies were excluded, which resulted in 2,908 articles being discarded. Finally, the remaining 21 articles were assessed for suitability, of which 15 publications fulfilled the inclusion and quality assessment criteria (Figure 1). All of these 15 studies were conducted in four countries: the UK (six), Brazil (four), the USA (three), and France (two).

\section{Quality assessment}

The results of the application of the quality assessment criteria were that one of the selected studies fulfilled eleven criteria, ${ }^{20}$ one met ten criteria, ${ }^{21}$ four met nine criteria, ${ }^{22-25}$ four met eight criteria, ${ }^{26-29}$ three met seven criteria, ${ }^{30-32}$ and two met six criteria. ${ }^{33,34}$ Only eight studies had obtained ethical approval. Two studies reported that ethical approval was not required, and five did not clearly state whether ethical approval had been obtained or not.

\section{Research methods used in selected studies}

Two primary methods were used within the selected studies: retrospective and prospective. Six were retrospective studies, of which five studies were conducted by reviewing incident reports, ${ }^{20,21,26,33,34}$ and one was conducted by reviewing patients' charts. ${ }^{32}$ By contrast, eight were prospective studies, of which seven used the direct observation method for the dispensary team, ${ }^{22-24,27,28,30,35}$ one was conducted by used face-to-face interviews with the dispensary team to investigate factors

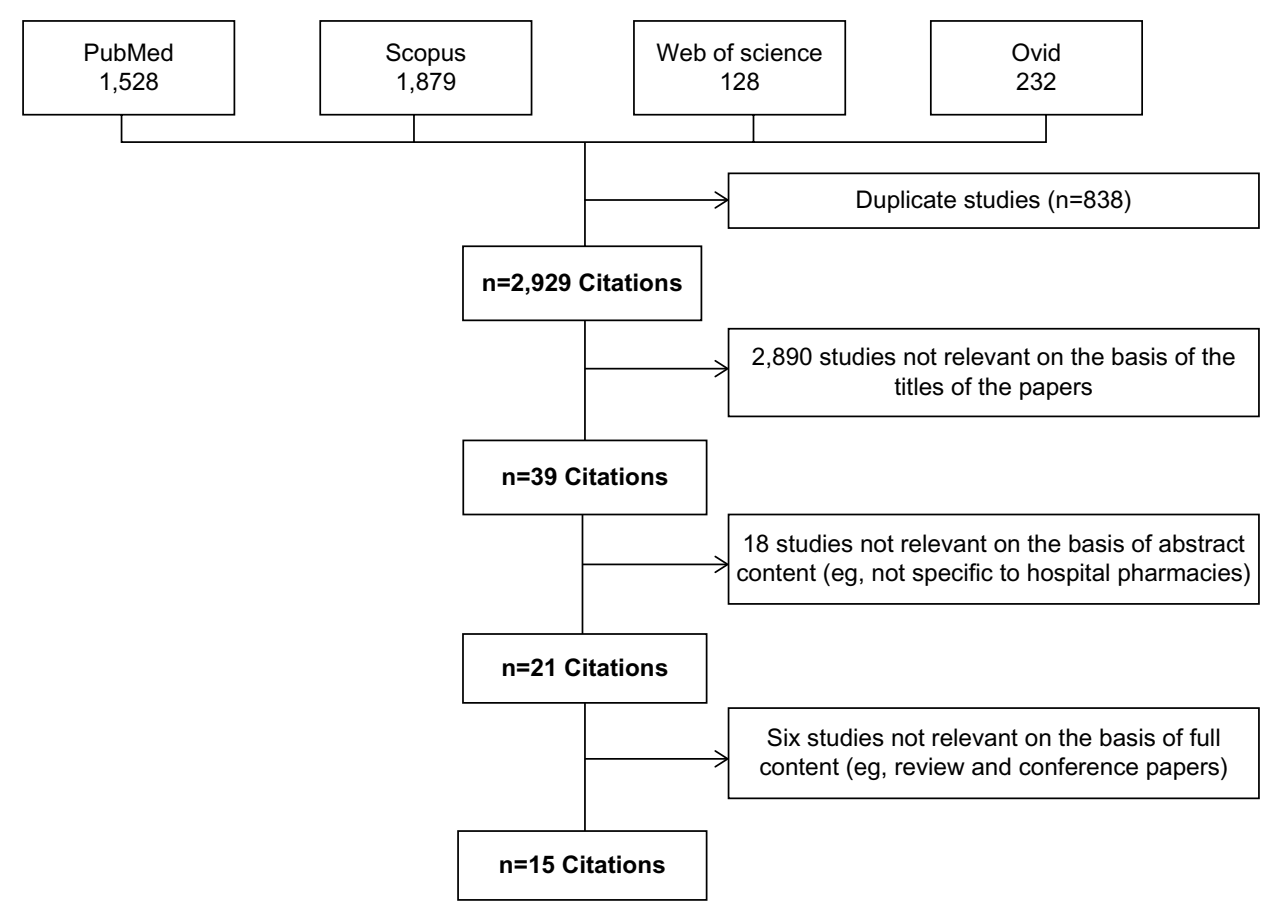

Figure I Summary of the literature search. 
associated with labeling errors. ${ }^{33}$ Furthermore, one study used a mixed method approach; the first part focused on observation to detect dispensing error types, and the second part involved interviewing the dispensary team to investigate the causes of dispensing errors. ${ }^{25}$ All Brazilian studies employed direct observation to investigate dispensing errors. By contrast, the majority $(80 \%)$ of UK studies relied on retrospectively reviewing incident reports. A brief description of these studies is presented in Table 1.

\section{Definition of dispensing errors}

None of the six studies reviewed in this paper define the term "dispensing error". However, definitions of dispensing errors were varied in the other published studies ${ }^{20,22,23,25,27,30,35}$ (Table 2). The definitions given in these studies are very similar; for example, the definition of a dispensing error is described as a discrepancy between the prescribed medication and actual medicine dispensed by the pharmacy. Some studies $^{20,24}$ use other definitions to distinguish between a dispensing error that is intercepted before the medicine leaves the pharmacy and after the medicine leaves the pharmacy. The errors detected after the medicines left the pharmacy were defined as unprevented (undetected) dispensing errors, and errors that were detected and reported before the medicines left the pharmacy were defined as prevented (detected or near miss[es]) dispensing errors.

\section{Incidence of dispensing errors}

This review identifies that there is variation in the rates of dispensing errors reported, determined as the number of dispensing errors divided by the number of dispensed items. The dispensing error rate detected using the prospective observation method ${ }^{22-24,27,28,30,35}$ is between $0.79 \%$ and $33.5 \%$. By contrast, in just two retrospective studies of incident reports, ${ }^{20,21}$ the rate of dispensing errors was reported as being between $0.0147 \%$ and $0.13 \%$, with more prevented dispensing error rates than unprevented dispensing error rates. In a study by James et $\mathrm{al},{ }^{20}$ which reviewed incident report to identify dispensing errors, the rate of prevented dispensing errors is given as $0.13 \%$ and $0.016 \%$ for unprevented dispensing errors. By contrast, in Cina's study, ${ }^{24}$ which used the observation method to detect dispensing errors, the rate of prevented dispensing errors was found to be $2.9 \%$, and $0.57 \%$ for unprevented dispensing errors.

\section{Dispensing error types}

In the identified published studies of dispensing errors, several categories were used to classify the different types of errors that occur during the dispensing process. Fourteen reviewed studies classified dispensing errors. ${ }^{20-32,34}$ All of the studies that identified the types of dispensing errors (14/14) reported that dispensing the wrong medicine was one of the most common error types. Other frequent dispensing errors reported in these studies include dispensing the wrong drug strength (11/14) and dispensing the wrong dosage form (9/14). Table 3 shows the types of dispensing errors cited in the identified published studies.

\section{Potential risk of dispensing errors}

In the reviewed studies, various categories were employed to evaluate the potential risks of dispensing errors. Six identified studies ${ }^{20,21,23,24,26,34}$ assessed the potential risks of dispensing errors. The majority of the dispensing errors in the reviewed papers were of minor clinical significance or caused no harm. However, some cases were serious and could have caused death; for example, a pharmacist dispensed an incorrect dose of verapamil $240 \mathrm{mg}$ instead of $40 \mathrm{mg}$ to an 86-year-old woman. ${ }^{23}$

\section{Factors associated with dispensing errors}

Only five identified published studies discussed contributing factors associated with dispensing errors. ${ }^{20,21,25,26,33}$ The factors most commonly associated with dispensing errors were high workload, low staff numbers, mix-up of look-alike/sound-alike drugs, and dispensary staff's lack of knowledge/experience. More contributing factors are presented in Table 4.

\section{Discussion}

Identifying types of dispensing errors and factors contributing to these errors is the first step in drawing up strategies to reduce such occurrences. The aim of this study was to review studies conducted in hospital pharmacies to identify the incidence and/or types and/or factors contributing to the occurrence of dispensing errors. To the best of the researchers' knowledge, no previous systematic review has focused on dispensing errors in hospital pharmacies only. This systematic review identified 15 studies carried out in just four different countries. The majority of these studies focus on dispensing error types only, and few studies analyze the severity of the errors, contributing factors, or strategies used to reduce dispensing errors.

Reviewing incident reports retrospectively and direct observation methods were the most commonly employed methods of investigating dispensing errors. All of the Brazilian and French studies used the observational method, 


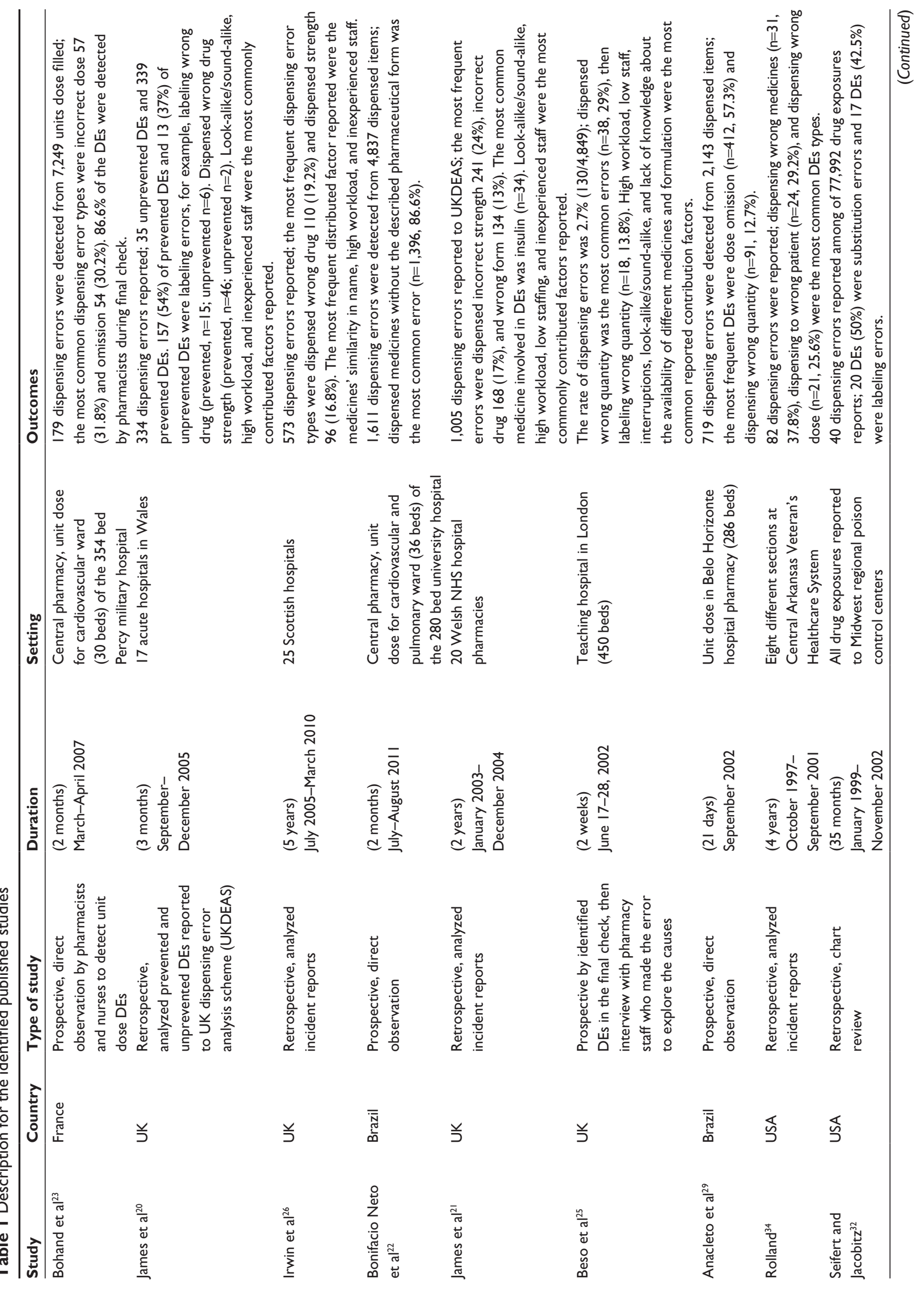




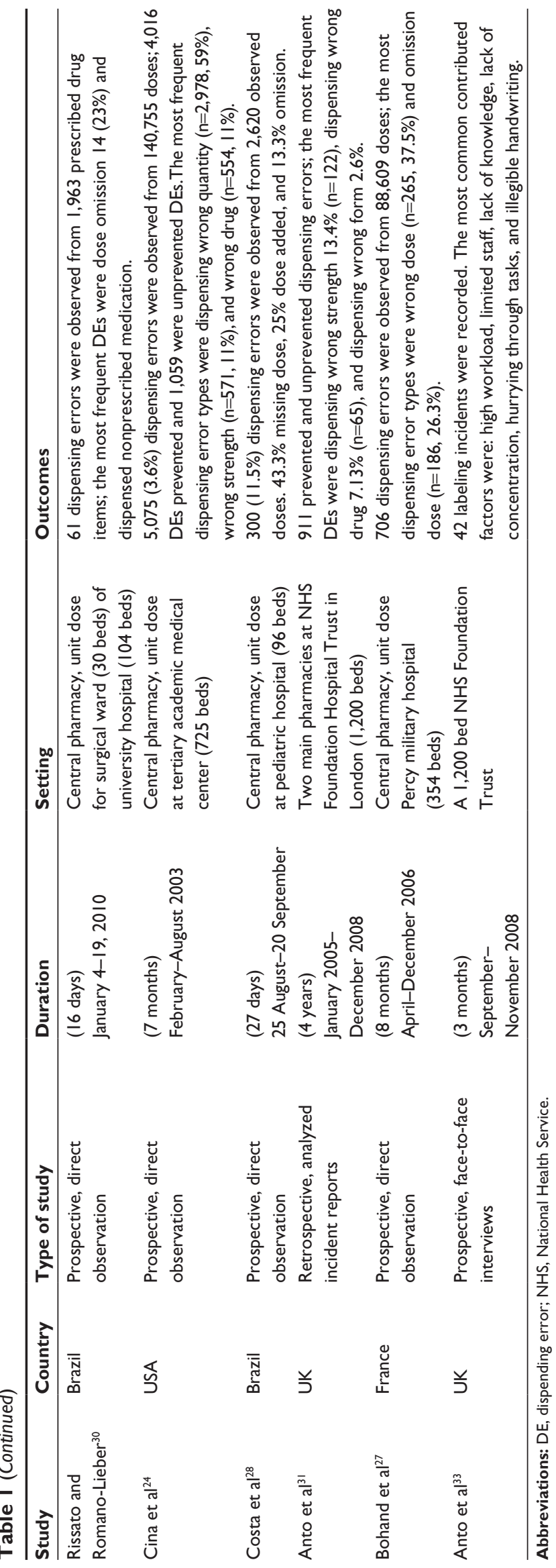

while the majority of the UK studies used incident reports. This suggests that reporting on medication errors in the UK is a more common and organized practice. The NRLS began recording such incidents in $2004 .{ }^{37}$ The NRLS help to provide rich data regarding received medication error reports, which contributes to the information needed to help identify the nature and cause of medication errors. Reporting dispensing errors and near-miss errors is an important strategy to build a safety culture and aid learning from previous errors. In order to encourage reporting, implementing a nonpunitive environment as well as robust facilities to facilitate the reporting process is important. ${ }^{38}$

In the identified published studies, a number of definitions are provided, all of which agree that a dispensing error is a "discrepancy between the prescription and dispensed medicines", though some studies add that it can also mean a "discrepancy between the modification made by a pharmacist to the prescription and dispensed medicines". ${ }^{20,22-25,27,30,35}$ However, some selected studies do not define the term "dispensing error". This raises certain questions for the researcher; for instance, whether the errors discussed in those studies include those made by nurses when dispensing medicines in a ward environment.

It was observed that the rates of dispensing errors were reported in all studies that used the observation method and that the rates of these were relatively high. By contrast, just two studies that employed a review of incident reports described the rate of dispensing errors; the rates are not presented in other studies usually because the total number of dispensed items is unknown. The rate of dispensing errors reported in the selected Brazilian studies ${ }^{22,28,29}$ is very high (11.5\%-33.5\%), compared with other selected studies in the UK, the USA, and France (3.6\%-0.016\%). This variation in dispensing error rate might be due to differences in the dispensing system, research methods, or the dispensing error classification used in the Brazilian studies..$^{30}$ For example, in one Brazilian study, ${ }^{22} \sim 87 \%$ of the dispensing errors were related to dispensed medicine, with no description of the dosage form. This type of dispensing error is not present in the categories included in other studies.

The most common error types reported in the selected studies are: dispensing the wrong drug, dispensing the wrong strength, dispensing the wrong quantity, and omission of items. Omitted dose is the most common dispensing error type in studies focusing on the identification of errors in dispensed items for inpatients (unit dose). ${ }^{23,28-30}$ Various categories are employed in the selected studies; some of these classifications are comprehensive, such as Beso's, ${ }^{25}$ and others ${ }^{22-24,27,30}$ focus 
Table 2 Definition of dispensing errors

\begin{tabular}{|c|c|c|}
\hline Term & Definition & References \\
\hline \multirow[t]{20}{*}{ Dispensing error } & "A discrepancy between the interpretable written prescription, including & 23 \\
\hline & modifications made by a pharmacist following contact with the physician or in & \\
\hline & accordance with pharmacy policy, and the contents of the medication cassette". & \\
\hline & "Deviation from a written prescription/medication order, including pharmacists' & 20 \\
\hline & written endorsements, occurring during the dispensing process of selecting and & \\
\hline & assembling medication (drug/content errors), generating and affixing dispensing & \\
\hline & labels (labeling errors) and issue of dispensed products to patients (issue errors)". & \\
\hline & $\begin{array}{l}\text { "Discrepancy between the prescribed medication and the content dispensed } \\
\text { by the pharmacy". }\end{array}$ & 22 \\
\hline & $\begin{array}{l}\text { "A deviation from an interpretable written prescription or medication order, } \\
\text { including written modifications to the prescription made by a pharmacist } \\
\text { following contact with the prescriber or in compliance with pharmacy policy". }\end{array}$ & 25 \\
\hline & "Discrepancy between the written instruction found on the prescription order & 35 \\
\hline & form and the accomplishment of this instruction by the pharmacy when the & \\
\hline & drug was dispensed to the wards or hospital services". & \\
\hline & "Any deviation from the written or oral prescription, including written modifications & 30 \\
\hline & by the pharmacist following contact with the prescriber or in compliance with & \\
\hline & pre-established norms and protocol, and any deviation from the stipulations of the & \\
\hline & appropriate regulatory agencies or norms was considered a drug-dispensing error". & \\
\hline & "Any discrepancy between dispensed medications and physician orders. & 24 \\
\hline & Any deviation from standard pharmacy policies". & \\
\hline & "Any discrepancy between the original or modified approved written & 27 \\
\hline & prescription, and the contents of the medication cassette". & \\
\hline Unprevented dispensing incidents & "Dispensing errors detected after the medication has left the pharmacy". & 20,24 \\
\hline \multirow[t]{2}{*}{ Prevented dispensing incidents } & "Dispensing errors detected during the dispensing process before the & 20,24 \\
\hline & medication had left the pharmacy”. & \\
\hline
\end{tabular}

on content errors and do not consider labeling errors. This may be because these studies focus on identifying dispensing errors in unit dose systems; however, labeling errors can have severe risks. For instance, a label with a wrong patient name might cause medicine to be given to a wrong patient.
The most common contributing factors identified in the reviewed studies were: mix-up of look-alikes/soundalikes, high pharmacy workloads, low staff numbers, inexperienced staff, and rushing to complete tasks. Only five of the selected studies discuss contributing factors: three

Table 3 Types of dispensing error

\begin{tabular}{|c|c|c|c|c|c|c|c|c|c|c|c|c|c|c|}
\hline Reference & 23 & 20 & 26 & 22 & 21 & 25 & 35 & 34 & 32 & 30 & 24 & 28 & 33 & 27 \\
\hline \multicolumn{15}{|l|}{ Content errors } \\
\hline Wrong medicine dispensed & $\mathrm{X}$ & $\mathrm{X}$ & $x$ & $\mathrm{X}$ & $\mathrm{X}$ & $\mathrm{X}$ & $x$ & $\mathrm{X}$ & $x$ & $\mathrm{X}$ & $\mathrm{X}$ & $\mathrm{X}$ & $\mathrm{X}$ & $\mathrm{X}$ \\
\hline Wrong drug strength dispensed & $\mathrm{X}$ & $\mathrm{X}$ & $\mathrm{X}$ & $\mathrm{X}$ & $\mathrm{X}$ & $\mathrm{X}$ & $\mathrm{X}$ & $\mathrm{X}$ & & $\mathrm{X}$ & $X$ & & $\mathrm{X}$ & \\
\hline Wrong dosage form dispensed & $X$ & & $X$ & & $X$ & $X$ & & & & $X$ & $X$ & $X$ & $X$ & $X$ \\
\hline Expired medicine dispensed & & & $\mathrm{X}$ & & & & & & & & & & & $X$ \\
\hline Omission of item & $\mathrm{X}$ & & $\mathrm{X}$ & $\mathrm{X}$ & & $X$ & $\mathrm{X}$ & & & $\mathrm{X}$ & & $\mathrm{X}$ & & $\mathrm{X}$ \\
\hline Wrong quantity dispensed & & $\mathrm{X}$ & & $\mathrm{X}$ & $\mathrm{x}$ & $\mathrm{X}$ & & & & & $\mathrm{X}$ & $\mathrm{X}$ & & \\
\hline Other content error & $\mathrm{X}$ & & X & $\mathrm{X}$ & & & & $\mathrm{X}$ & & $\mathrm{X}$ & & $\mathrm{X}$ & & \\
\hline \multicolumn{15}{|l|}{ Labeling errors } \\
\hline Wrong patient name & & $\mathrm{X}$ & $\mathrm{X}$ & & $\mathrm{X}$ & $\mathrm{X}$ & & & & & & $\mathrm{X}$ & & \\
\hline Wrong medicine name & & & & & $\mathrm{X}$ & & & & $\mathrm{x}$ & & & & & \\
\hline Wrong medicine strength & & & & & $\mathrm{X}$ & & & & & & & $\mathrm{X}$ & & \\
\hline \multicolumn{15}{|l|}{ Wrong frequency } \\
\hline Wrong dosage form & & & & & & & & & $x$ & & & & & \\
\hline \multicolumn{15}{|l|}{ Wrong date } \\
\hline Wrong instructions & & & $\mathrm{X}$ & & & $\mathrm{X}$ & & & $x$ & & & & & \\
\hline \multicolumn{15}{|l|}{ Completely wrong label } \\
\hline \multicolumn{15}{|l|}{ Incomplete information } \\
\hline Other labeling error & & $\mathrm{X}$ & $\mathrm{X}$ & & $\mathrm{X}$ & $X$ & $\mathrm{X}$ & & & & & 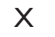 & & \\
\hline Other error & & & & & & & & & $x$ & & & $\mathrm{X}$ & & \\
\hline
\end{tabular}

Note: $X$ denotes inclusion in selected studies. 
Table 4 Dispensing error contributed factors

\begin{tabular}{|c|c|c|c|c|c|}
\hline Reference & 20 & 26 & 21 & 25 & 33 \\
\hline \multicolumn{6}{|l|}{ Work environment } \\
\hline High workload & 70 & 29 & $14 \mid$ & 22 & $x$ \\
\hline Low staffing & 38 & 14 & 74 & 13 & $x$ \\
\hline Distraction/interruption & 30 & II & & 14 & $x$ \\
\hline Noise & & 2 & & & \\
\hline Protocols not followed & & II & & 2 & \\
\hline Dispensary design & & & & 4 & \\
\hline Lone worker & 9 & & 10 & & \\
\hline Time of day & 29 & & & & $x$ \\
\hline \multicolumn{6}{|l|}{ Product } \\
\hline Look-alike/sound-alike drug name & 37 & 30 & 233 & 9 & \\
\hline Similarity packaging & & 3 & & & \\
\hline Poor labeling by manufacturer & & 2 & & & \\
\hline \multicolumn{6}{|l|}{ Team } \\
\hline Inexperienced staff & 73 & 26 & 114 & 7 & $x$ \\
\hline Communication problem & 6 & & 43 & I & \\
\hline Loss of concentration/fatigue & & 2 & & 12 & \\
\hline Low moral & & & & 2 & \\
\hline Urgent deadline/hurrying through tasks & 22 & 4 & 49 & 12 & $x$ \\
\hline \multicolumn{6}{|l|}{ Task } \\
\hline Complex prescription & 6 & & 2 & & \\
\hline Illegible handwriting & & 4 & & & $x$ \\
\hline Careless checking & & 14 & & & \\
\hline Unfamiliarity with task & & 9 & & 5 & \\
\hline Patient demanding/aggression & & 5 & & & \\
\hline
\end{tabular}

Notes: The numbers denote how many times these contributing factors had been reported in the study. $X$ denotes a mention of contributing factors but without numbers of reported incidents.

studies $^{20,21,26}$ gathered information about contributing factors from incident reports, and two studies ${ }^{25,33}$ did the same by interviewing dispensary teams to discover contributing factors. None of the selected studies relied on observation to uncover contributing factors.

These results indicate certain limitations in the methods employed to investigate dispensing errors. For example, the incident report approach does not provide a rate of incident occurrence, and so the total number of reported dispensing errors is uncertain, as some errors were not indicated, or indicated but not reported.$^{20} \mathrm{In}$ addition, some of the incident reports did not indicate contributing factors. By contrast, all of the observation studies reported the exact incident rate, but no information was given about contributing factors. While qualitative methods, such as interviews, provided an insight into contributing factors, these studies failed to investigate the types of dispensing errors. In order to resolve these limitations, a mixed methods approach is required for use in future studies if they are to investigate both the type of error and contributing factors; the existing studies that utilized a mixed methods approach provided more and accurate details regarding the nature of errors and the contributing factors. ${ }^{25,39}$ The unique contribution of the combination method research is that it allows for the integration of results from more than one component of a study. ${ }^{40}$

Finally, an investigation into dispensing error types and contributing factors in hospital pharmacies is very useful to set strategies to improve patient safety. The Institute for Safe Medication Practices ${ }^{41}$ recommend some strategies to reduce dispensing errors that are linked to medicines with similar names such as storage in different locations and coloring coding and/or using tall man letter. The NRLS published a guideline booklet ${ }^{7}$ which aims to minimize contributing factors to dispensing errors through well-designed pharmacies. Moreover, educating the dispensary team about observed errors in order to avoid future errors can be an effective strategy to enhance patient safety. ${ }^{23}$

\section{Strengths and limitations of this study}

To our knowledge, this study is the first systematic review of dispensing errors in hospital pharmacies only. An exhaustive literature search was undertaken which identified all studies fulfilling the inclusion criteria. A quality assessment was performed for each study included in the review.

The limitation of this review is that it is restricted to studies published in the English language, so some studies in different languages are not covered. Another limitation is that "gray literature" (unpublished reports) was not surveyed. These reports may contain useful data, but in the absence of peer-review and concerns about impartiality, their relevance cannot be guaranteed.

\section{Conclusion}

Many existing studies identify and discuss dispensing errors in community pharmacies, but few studies have investigated this phenomenon in a hospital pharmacy environment. This review identified just 15 studies conducted in hospital pharmacies. The majority of these studies focus on investigating the types of dispensing errors, and few discussed the factors contributing to these or the strategies used to reduce dispensing errors. Although no evidence was apparent from the review about the impact of dispensing errors on the patient, dispensing errors may lead to a number of situations including inconvenience, confusion, and inappropriate therapy which may result in some degree of harm in some cases. The results of this review highlight the fact that dispensing the wrong medicine is a common dispensing error type, as it is indicated in all selected studies. Future studies investigating dispensing errors should use a mixed methods approach to investigate the contributing factors associated with dispensing errors and explore the strategies employed for reducing these errors. In 
addition, clear definitions and comprehensive classifications of dispensing error types are still needed.

\section{Disclosure}

The authors report no conflicts of interest in this work.

\section{References}

1. Milch CE, Salem DN, Pauker SG, Lundquist TG, Kumar S, Chen J. Voluntary electronic reporting of medical errors and adverse events. J Gen Intern Med. 2006;21(2):165-170.

2. Cousins DH, Gerrett D, Warner B. A review of medication incidents reported to the National Reporting and Learning System in England and Wales over 6 years (2005-2010). Br J Clin Pharmacol. 2012;74(4):597-604.

3. NHS. [webpage on the Internet]. Organisation Patient Safety Incident Reports - Data Workbooks; 2014. Available from: http://www.nrls.npsa. nhs.uk/patient-safety-data/organisation-patient-safety-incident-reports/ directory/. Accessed December 9, 2015.

4. Kelly WN. Pharmacy: What It Is and How It Works. Boca Raton, FL: CRC Press; 2011.

5. HSCIC. Prescriptions Dispensed in the Community: England 2002-12. Vol 2015. Health and Social Care Information Centre; 2013.

6. Aitken M, Kleinrock M, Lyle J, Caskey L. Medicine Use and Shifting Costs of Healthcare: A Review of the Use of Medicines in the United States in 2013. Parsippany, NJ: IMS Institute for Healthcare Informatics. IMS Health; 2014.

7. NPSA. Design for Patient Safety: A Guide to the Design of the Dispensing Environment. London: NPSA; 2007.

8. Flynn EA, Barker KN, Carnahan BJ. National observational study of prescription dispensing accuracy and safety in 50 pharmacies. $J \mathrm{Am}$ Pharm Assoc (Wash.). 2003;43(2):191-200.

9. NPSA. Safety in Doses: Improving the Use of Medicines in the NHS. London: NPSA; 2007.

10. Lisby M, Nielsen LP, Mainz J. Errors in the medication process: frequency, type, and potential clinical consequences. Int J Qual Health Care. 2005;17(1):15-22.

11. Alakhali KM, Ansari SMA, Alavudeen SS, Ivhan NA. Medication errors at the outpatient pharmacy in a hospital in Aseer region, Kingdom of Saudi Arabia. Atencion Farmaceut. 2014;16(2):144-146.

12. Karthikeyan M, Lalitha D. A prospective observational study of medication errors in the general medicine department in a tertiary care hospital Drug Metabol Drug Interact. 2013;28(1):13-21.

13. Kirke C. Medication safety in hospitals. Ir Med J. 2009;102(10): 339-341.

14. Franklin BD, Reynolds M, Sadler S, et al. The effect of the electronic transmission of prescriptions on dispensing errors and prescription enhancements made in English community pharmacies: a naturalistic stepped wedge study. BMJ Qual Saf. 2014;23(8):629-638.

15. Royal Pharmaceutical Society. Near Miss Error Guide. London: Royal Pharmaceutical Society; 2015. Available from: http://www.rpharms. com/non-member/non-member.asp. Accessed May 5, 2015.

16. Alsulami Z, Conroy S, Choonara I. Medication errors in the Middle East countries: a systematic review of the literature. Eur J Clin Pharmacol. 2013;69(4):995-1008.

17. Ghaleb MA, Barber N, Franklin BD, Yeung VW, Khaki ZF, Wong IC. Systematic review of medication errors in pediatric patients. Ann Pharmacother. 2006;40(10):1766-1776.

18. James KL, Barlow D, McArtney R, Hiom S, Roberts D, Whittlesea C. Incidence, type and causes of dispensing errors: a review of the literature. Int J Pharm Pract. 2009;17(1):9-30.

19. Allan EL, Barker KN. Fundamentals of medication error research. Am J Hosp Pharm. 1990;47(3):555-571.

20. James KL, Barlow D, Burfield R, Hiom S, Roberts D, Whittlesea C. Unprevented or prevented dispensing incidents: which outcome to use in dispensing error research? Int J Pharm Pract. 2011;19(1):36-50.
21. James KL, Barlow D, Burfield R, Hiom S, Roberts D, Whittlesea C. A study of unprevented dispensing incidents in Welsh NHS hospitals. Int J Pharm Pract. 2008;16(3):175-188.

22. Bonifacio Neto AA, de Matos VTG, Toffoli Kadri MC. Evaluation of drug-dispensing errors at the internal medicine of an University Hospital. Latin Am J Pharm. 2013;32(1):26-30.

23. Bohand X, Simon L, Perrier E, Mullot H, Lefeuvre L, Plotton C. Frequency, types, and potential clinical significance of medicationdispensing errors. Clinics. 2009;64(1):11-16.

24. Cina JL, Gandhi TK, Churchill W, et al. How many hospital pharmacy medication dispensing errors go undetected? Jt Comm J Qual Patient Saf/Jt Comm Resour. 2006;32(2):73-80.

25. Beso A, Franklin BD, Barber N. The frequency and potential causes of dispensing errors in a hospital pharmacy. Pharm World Sci. 2005;27(3): 182-190.

26. Irwin A, Ross J, Seaton J, Mearns K. Retrospective analysis of DATIX dispensing error reports from Scottish NHS hospitals. Int J Pharm Pract. 2011;19(6):417-423.

27. Bohand X, Aupee O, Le Garlantezec P, Mullot H, Lefeuvre L, Simon L. Medication dispensing errors in a French military hospital pharmacy. Pharm World Sci. 2009;31(4):432-438.

28. Costa LA, Valli C, Alvarenga AP. Medication dispensing errors at a public pediatric hospital. Rev Lat Am Enfermagem. 2008;16(5): $812-817$.

29. Anacleto TA, Perini E, Rosa MB, Cesar CC. Medication errors and drug-dispensing systems in a hospital pharmacy. Clinics (Sao Paulo, Brazil). 2005;60(4):325-332.

30. Rissato MAR, Romano-Lieber NS. Drug dispensing errors at a university hospital in Brazil. Latin Am J Pharm. 2013;32(1):60-66.

31. Anto B, Barlow D, Oborne CA, Whittlesea C. Incorrect drug selection at the point of dispensing: a study of potential predisposing factors. Int J Pharm Pract. 2011;19(1):51-60.

32. Seifert SA, Jacobitz K. Pharmacy prescription dispensing errors reported to a regional poison control center. J Toxicol Clin Toxicol. 2002;40(7):919-923.

33. Anto B, Barlow D, Oborne A, Cape A, Vlassoff A, Whittlesea C. Dispensing-label errors in hospital: types and potential causes. Int $J$ Pharm Pract. 2010;18(2):122-124.

34. Rolland P. Occurrence of dispensing errors and efforts to reduce medication errors at the Central Arkansas Veteran's Healthcare System. Drug Saf. 2004;27(4):271-282.

35. Anacleto TA, Perini E, Rosa MB, Cesar CC. Drug-dispensing errors in the hospital pharmacy. Clinics (Sao Paulo, Brazil). 2007;62(3): 243-250.

36. Roberts DE, Spencer MG, Burfield R, Bowden S. An analysis of dispensing errors in NHS hospitals. Int J Pharm Pract. 2002;10(S1): R6-R6.

37. Scobie S, Thomson R, Cook A, Carthey J. Building a Memory: Preventing Harm, Reducing Risks and Improving Patient Safety. London, England: National Patient Safety Agency; 2005.

38. Brady M. How to improve patient care by learning from mistakes: Mike Brady explains why healthcare services need non-punitive working cultures to ensure that staff can identify and understand errors in practice. Emerg Nurse. 2013;20(9):32-35.

39. Ashcroft D, Lewis P, Tully M, et al. Prevalence, nature, severity and risk factors for prescribing errors in hospital inpatients: prospective Study in 20 UK Hospitals. Drug Saf. 2015;38(9):1-11.

40. Protheroe J, Bower P, Chew-Graham C. The use of mixed methodology in evaluating complex interventions: identifying patient factors that moderate the effects of a decision aid. Fam Pract. 2007;24(6): 594-600.

41. The Institute for Safe Medication Practices. What's in a Name? Ways to Prevent Dispensing Errors Linked to Name Confusion. Horsham, PA: The Institute for Safe Medication Practices; 2002. Available from: http://www.ismp.org/newsletters/acutecare/articles/20020612_2.asp. Accessed October 10, 2015 
Integrated Pharmacy Research and Practice

Dovepress

\section{Publish your work in this journal}

Integrated Pharmacy Research and Practice is an international, peer-reviewed, open access, online journal, publishing original research, reports, reviews and commentaries on all areas of academic and professional pharmacy practice. This journal aims to represent the academic output of pharmacists and pharmacy practice with particular focus on integrated care. All papers are carefully

peer reviewed to ensure the highest standards as well as ensuring that we are informing and stimulating pharmaceutical professionals. The manuscript management system is completely online and includes a very quick and fair peer-review system, which is all easy to use. Visit http://www.dovepress.com/ testimonials.php to read real quotes from published authors.

Submit your manuscript here: http://www.dovepress.com/integrated-pharmacy-research-and-practice-journal 